\title{
Review of "Qeios"
}

Gug lielmo Bonaccorsi ${ }^{1}$

1 University of Florence

fantastic! it needs no other words, it is complete and exhaustive 